\title{
Visual search under conditions of very rapid sequential input rates'
}

\author{
CHARLES W. ERIKSEN ${ }^{2}$ AND TERRY J. SPENCER \\ UNIVERSITY OF ILLINOIS
}

\begin{abstract}
The purposes of the present experiment were to provide information on rate of information processing in visual perception and to determine the degree to which the "sequential blanking" effect found by Mayzner, Tresselt, and Cohen (1966) constituted a limitation on rapid sequential input rates. A 10-channel tachistoscope was employed that permitted controlled durations of each of the 10 channels and the 9 interchannel intervals. The $S$ 's task was one of visual search or detection in which he searched for a target letter among noise letters. A temporal interval forced-choice procedure was used. In addition to varying the rate at which letters were sequentially presented, various irregular temporal spatial orders of presentation of the letter sequences were employed and the direction and orientation of the display in the visual field was varied as was also the spacing between adjacent stimuli. No evidence of "sequential blanking" was found either in terms of the detection criterion or in the Ss' phenomenal reports. Detection performance was as good at a rate of $21 / 2$ msec per letter as it was at a rate of $30 \mathrm{msec}$ per letter.
\end{abstract}

Little information is available concerning visual information processing under conditions of rapid sequential input. Attempts to determine encoding rate in visual perception have typically used one or the other of two techniques. The first of these is to determine the exposure duration necessary to correctly identify one, two, three, etc. letters or digits presented simultaneously in a visual display. A second method that has been introduced by Estes and his associates (Estes \& Taylor, 1964; 1966; Estes \& Wessel, 1966) consists of presenting a 4 by 4 matrix containing noise and target letters for a brief duration with the S's task the detection of the target (s). By varying the number of noise letters contained in the display estimates are obtained as to the rate $S$ processes the display elements in the search for the target.

With modern electronic equipment a different approach to the problem is possible. Instead of having to estimate processing rate by presenting simultaneously displays containing different amounts of information, it is possible to present each item in a display individually and in sequence at different rates. Mayzner and his associates (Mayzner, Tresselt, \& Cohen, 1966; Mayzner, Tresselt, \& Helfer, 1967) have reported some preliminary findings employing a computer based CRT display system. Their experimentation has not as yet been directed to answering the perceptual or encoding rate problem but instead has focused on a phenomenon they have termed sequential blanking. If a sequence of letters is presented in a horizontal array at a rate in the range of approximately 10 to $20 \mathrm{msec}$ per letter and interval between letters, the first half of these letters will not be perceived if the display order is irregular. While this sequential blanking does not occur with all irregular orders it does suggest some limitations, if not upon the input rate of perception, at least upon this methodology for studying encoding or processing rate.

It seems likely that this blanking effect obtained by Mayzner et al (1967) may be an instance of apparent movement as they themselves have noted. Their stimulus presentation is similar to the metacontrast experimental arrangement of Alpern (1953) where a light stimulus is subsequently followed by two flanking lights. At certain interstimulus intervals (ISIs) the Ss report not seeing the center light. However, from attempts in our laboratory to repeat these observations, we have found it impossible to obtain the effect without the occurrence of apparent movement. While Ss will report not observing the central light phenomenally they invariably report under these ISIs that the two flanking lights appear to have moved out away from each other. Given a forced-choice procedure Ss can almost always detect whether the center light has been present or not due to the apparent movement that they observed in the flanking lights.

\section{EXPERIMENT 1}

It was one purpose of the present experiment to determine the degree to which sequential blanking would constitute a limitation on a methodology employing rapid sequential input rates in visual perception. A 10-channel tachistoscope was employed that permitted controlled durations of each of the 10 channels and the 9 interchannel intervals. The S's task was one of visual search or detection in which he searched for a target letter among noise letters. In addition to varying the irregular temporal spatial order of presentation of letter sequences, the foveal sensitivity where the different stimuli fell was controlled and direction and orientation of the display as well as spacing between adjacent stimuli was varied.

A second and equally important aim of the present experiment was to provide information upon the rate of visual information processing. Sperling (1963) has concluded that for up to four or five letters processing occurs at a rate of approximately $10 \mathrm{msec}$ per letter. Similar time values have been obtained by Scharf, Zamansky, and Brightbill (1966). From these estimates of the processing rate the range of 10 to $20 \mathrm{msec}$ per letter and interval between letters where Mayzner et al (1967) obtain sequential blanking should be adequate for the processing of each letter in a sequential input.

\section{Subjects}

\section{METHOD}

Five students, two female, with normal or corrected to normal vision served as paid volunteers.

Apparatus and Stimuli

A 10-channel tachistoscope was especially designed to attack the experimental problem. Ten Sylvania fluorescent lamps (F4 T5/CWX) were individually mounted in light proof boxes which were then attached to the surface of a sheet metal drum and equally spaced around the circumference. From each light proof box a flexible fiber optic light guide $1 / 8$ in. inner diameter and 12 in. long extended to a machined fitting where each was coupled to a 1/4 in. diameter Lucite rod 6 in. long. The ends of the Lucite rods extended through the machined fitting and formed a circular pattern that subtended a diameter of $1.3 \mathrm{deg}$ of angle with the center of each light guide spaced $.42 \mathrm{deg}$ from the centers of the adjacent light guides. A separate light source projected at the center of the circular arrangement and was used to provide an $x$ fixation point of $.25 \mathrm{deg}$ of angle. The machined fitting provided for $35 \mathrm{~mm}$ slides to be placed immediately in front of the projecting ends of the 10 Lucite rods and the fixation light source. Additional space in the fitting provided for neutral density filters mounted as $35 \mathrm{~mm}$ slides.

The machined fitting was mounted at the end of a visual tunnel 18 in. sq and 30 in. long. At the S's end of the visual tunnel was a viewing hood and head rest. The distance from the S's eye to the $35 \mathrm{~mm}$ slide in front of the 10 light guides was 38 in. Figure 1 shows the display arrangement as it appeared to the S's view when all 10 lights were simultaneously fired. The 10 fields are numbered in Fig. 1 to permit identification. Also given in Fig. 1 are the physical sizes of the display in inches.

The lamps for the tachistoscope were fired with $300 \mathrm{~V} \mathrm{dc}$ and at all times had a subcritical heating voltage applied to them to insure reliability of firing. Ten Tektronic waveform generators were used to control the duration of the stimulus fields and nine pulse form generators controlled the ISIs between the fields. 


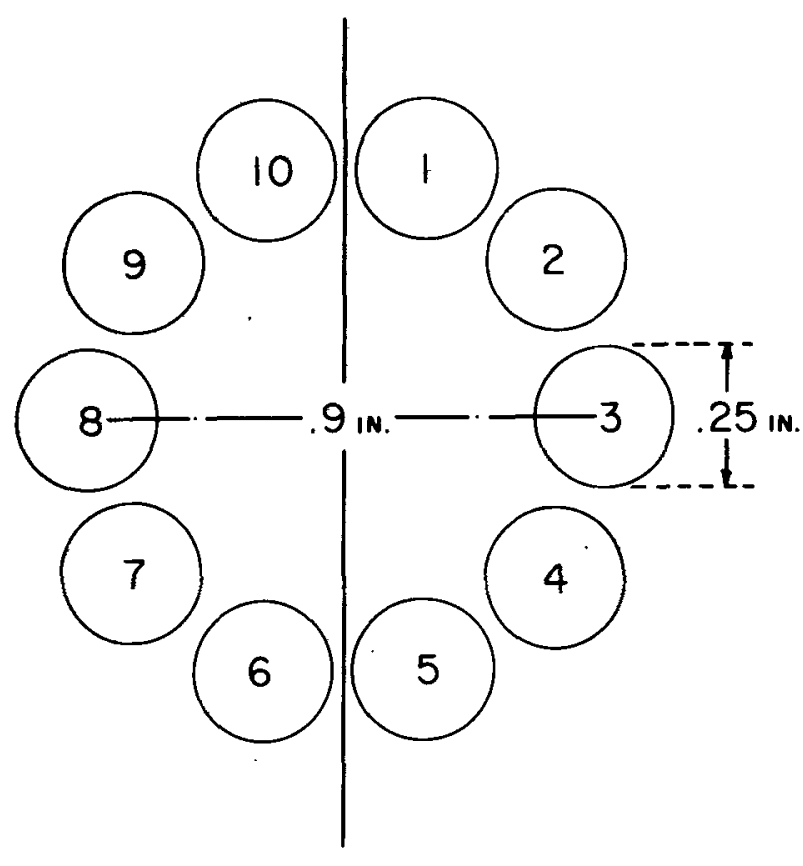

Fig. 1. Arrangement and physical size of the $\mathbf{1 0}$ fields of the tachistoscope as viewed by $\mathbf{S}$.

In order to vary the order and the number of the light fields presented to the $S$, a scrambler box was inserted in the circuit between the light sources and the timers. This consisted of a base in which were mounted 10 copper strips each connected to one of the field timers. The top of the scrambler was similarly constructed except that the 10 copper strips were at right angles to those in the base and were connected directly to the light source through transistorized switches. To complete the circuit between the base and the top of the scrambler 10 by 10 matrix peg boards were used. By placing metal pins in the appropriate holes in the peg boards any desired number, pattern, or order of presentation of the 10 stimulus fields could be obtained. Peg boards composing the different orders of stimulus presentation to be used in the experiment were constructed prior to the experiment and could be quickly inserted and removed between trials.

The stimulus slides for use in the tachistoscope were constructed by photographing white letters against a black background. A layout was so scaled that when the letters were placed in the indicated positions on the layout the appropriate location and reduction in letter size of the photograph was obtained. The fixation cross was provided in the center of the layout so that each slide contained its own fixation stimulus. The photographing of the stimuli was done using high contrast negative film (Polaroid 149L). As viewed by the $S$ illumination of one of the light fields presented a transilluminated letter having a contrast of better than $98 \%$ with the surrounding ground. The letters subtended .18 deg of visual angle and had a luminance of $.3 \mathrm{~mL}$.

\section{Procedure}

A two interval forced-choice procedure was used throughout the experiment. The stimul were the three capital letters $A, T$, and $U$, with $A$ designated the 'arget and the $T$ and $U$ as noise. Under the single letter condition one of the three letters was presented in either field $1,3,6$, or 9 of the tachistoscope for short duration and $500 \mathrm{msec} f$ llowing its offset the second letter appeared in one of the remaining three fields according to a random order. The $S$ was $r$ zquired to choose which of these two intervals had contained the $A$ (Ine interval always contained the $A$ and the other interval a $T \mathrm{cr}$ a $U$ equally often. Under the five letter condition a trial consisted of the presentation of five letters at each of the two tempral intervals. One interval always contained a random assortment of five of the two noise letters and the other interval always contained the target and four occurrences of the noise letters. Again the $S$ was required to choose which of the two intervals had contained the target.

For the five letter condition four spatial positions in the tachistoscopic fields were used for stimulus presentation. Spatial Position I consisted of tachistoscopic fields 1 through 5 (see Fig. 1), Position Il fields 3 through 7, III fields 6 through 10, and spatial Position IV fields $8,9,10,1$, and 2. For the two intervals on a forced-choice trial opposite spatial positions were employed. Thus on any given trial spatial Position I would be used for one of the forced-choice intervals with spatial Position III for the second interval, or spatial Position II would be paired with spatial Position IV. Which of the spatial fields occurred first on a trial as well as which contained the target letter $\mathbf{A}$ was counterbalanced.

$A$ second variable for the five letter condition involved the pattern sequence in which the five fields in a given spatial position presented their stimuli. Table 1 shows the temporal order in which the different fields came on for each of the five pattern sequences under each of the four spatial positions. As an example, under Sequence $A$ the stimuli occurred successively in the temporal order going in a clockwise direction through the fields of the tachistoscope. Sequence B was a sequence reported by Mayzner et al (1967) to produce pronounced sequential blanking, and Sequences C and D were other irregular sequences chosen for investigation. Sequence $\mathrm{E}$ differed from the other sequences in that the stimuli were spaced twice as far apart as for the other sequences. This was accomplished by using only every other field of the tachistoscope and a presentation of five letters described a complete circle of stimulation. Thus when spatial Position I was used for this sequence stimuli occurred in fields $1,3,5,7$, and 9 for one of the forced-choice intervals and in 2, 4,6, 8, and 10 for the other interval. The temporal sequence in which these spatial positions came on is shown in Table 1 and is the same as Sequence B except for the separation of stimuli. The use of two pattern sequences that were identical except for the spatial separation of the stimuli served to check upon the possibility that blanking effects were attributable to apparent movement.

All sequences were used with each spatial position equally of ten and the same sequence was used in both forced-choice intervals. For each sequence the target occurred an equal number of times in each of the five possible positions. For each $S$ there was a total of 200 trials with each of the five pattern sequences. In addition each S was given 96 trials under the single letter condition where the

Table 1

The Temporal Order in which Five Tachistoscopic Fields Present Stimuli under the Five Pattern Sequences for the Four Spatial Positions

\begin{tabular}{ccccccc}
\hline Spatial & Pattern & \multicolumn{5}{c}{ Temporal order } \\
position & sequence & 1 & 2 & 3 & 4 & 5 \\
\hline \multirow{3}{*}{ I } & A & 1 & 2 & 3 & 4 & 5 \\
& B & 4 & 2 & 5 & 1 & 3 \\
& C & 3 & 1 & 2 & 5 & 4 \\
D & 1 & 4 & 3 & 5 & 2 \\
& E & 7 & 3 & 9 & 1 & 5 \\
\hline \multirow{3}{*}{ II } & A & 3 & 4 & 5 & 6 & 7 \\
& B & 6 & 4 & 7 & 3 & 5 \\
& C & 5 & 3 & 4 & 7 & 6 \\
& D & 3 & 6 & 5 & 7 & 4 \\
& E & 9. & 5 & 1 & 3 & 7 \\
\hline \multirow{3}{*}{ III } & A & 6 & 7 & 8 & 9 & 10 \\
& B & 9 & 7 & 10 & 6 & 8 \\
& C & 8 & 6 & 7 & 10 & 9 \\
& D & 6 & 9 & 8 & 10 & 7 \\
& E & 2 & 8 & 4 & 6 & 10 \\
\hline \multirow{3}{*}{ IV } & A & 8 & 9 & 10 & 1 & 2 \\
& B & 1 & 9 & 2 & 8 & 10 \\
& C & 10 & 8 & 9 & 2 & 1 \\
& E & 4 & 10 & 6 & 8 & 2 \\
\hline
\end{tabular}


letter A occurred in each of the two forced-choice intervals 48 times.

Prior to beginning experimental sessions Ss were run several practice sessions during which an exposure duration for single letter presentations was determined that yjelded about $90 \%$ forced-choice accuracy. The exposure duration determined for each $\mathrm{S}$ during these practice sessions was then used as the exposure duration for each letter occurrence and also the duration of the interletter intervals in the five letter condition. These times ranged from $8 \mathrm{msec}$ to $20 \mathrm{msec}$ with three Ss requiring changes to shorter exposure durations within this range at counterbalanced points within the experiment.

During each experimental session each five letter sequence occurred an equal number of times, the order being randomized within the session. The single letter condition was presented in three sessions, one near the beginning, middle, and end of the experiment. There were 100 trials in each session except for the sessions in which the single letter condition was presented. Here there were 82 trials, 50 for the five letter condition and 32 for the single letter condition.

Subjects were instructed that the A would occur in one or the other of the two temporal intervals but not both. They were asked to respond to each trial indicating in which interval they thought the $A$ had occurred. Prior to each experimental session the $S$ was dark adapted for $7 \mathrm{~min}$ and then given several warm-up trials before beginning the experimental judgments.

\section{RESULTS AND DISCUSSION}

The first analyses were to determine whether under the five letter condition target detection varied as a function of the temporal order of occurrence of the target, its spatial position, or the pattern sequence in which it occurred. Per cent correct forced-choice scores for each $S$ were analyzed in a four-way analysis of variance (the three above mentioned variables plus $\mathrm{Ss}$ ). None of the main effects approached significance but the temporal order by spatial location interaction was of borderline significance $[F(12,48)=2.02, p>.05]$.

In Fig. 2 this interaction is presented graphically. Per cent correct decisions are plotted as a function of the temporal order in which the target occurred among the five stimuli for each of the four spatial locations of the 10 tachistoscopic fields. For spatial Locations I and III where the five letters were presented in tachistoscopic fields 1 through 5 and in fields 6 through 10 , detection of the $A$ was best when it was the fourth letter presented temporally and poorest when it was first or fifth. For spatial Positions II and IV most accurate detection occurred when the target was either the first or the last temporally presented letter and detection was poorest when the target was the fourth temporally.

The variation in detection accuracy as a function of the temporal order of occurrence of the target and whether the top, bottom, or right or left half of the circular display was used in presenting the five letters is not readily explainable. One possibility is that all 10 fields of the tachistoscope are not equal in terms of ease in perceptual identification and since it was not experimentally. feasible to test all possible sequences of fields with temporal order, certain tachistoscopic fields occurred disproportionately often with specific temporal orders. Thus if tachistoscopic field 6 was below average for perceptual accuracy and the pattern sequences disproportionately used field 6 for temporal order 4 for spatial Positions II and IV, the effect would be an interaction such as was obtained.

There is evidence that perceptual accuracy is not equal for all quadrants around a central fixation point. Keeley (1968) used a circular display centered on a central fixation point such as employed in this study and found that perceptual accuracy was poorest for positions corresponding to six o'clock. In Fig. 3 we have plotted detection accuracy for each of the 10 tachistoscopic fields. The results are very comparable to those obtained by Keeley (1968). Performance is best when the stimuli appear to the left and slightly above the fixation point and poorest when presented below and to the lower right or the left of the fixation point. An analysis of variance of the data in Fig. 3 shows them significant beyond the .05 level $[F(9,36)=2.4]$.

Although there is the expected variation in perceptual accuracy for the different field locations around the fixation point, this does not provide an unequivocal explanation for the interaction between spatial position and temporal order. If the most and the least accurate tachistoscopic field positions are determined for the different temporal orders for the four spatial locations no clear pattern emerges that would account for the obtained interaction. It would appear that the interaction must remain unexplained unless it is to be attributed to chance. This latter alternative is not unlikely considering the low level of significance and the number of comparisons that occurred in the four-way analysis of variance.

The next question to be asked of the data concerns the effect of rate of presentation upon detection accuracy. For the single letter condition where only one letter was presented and the forced-

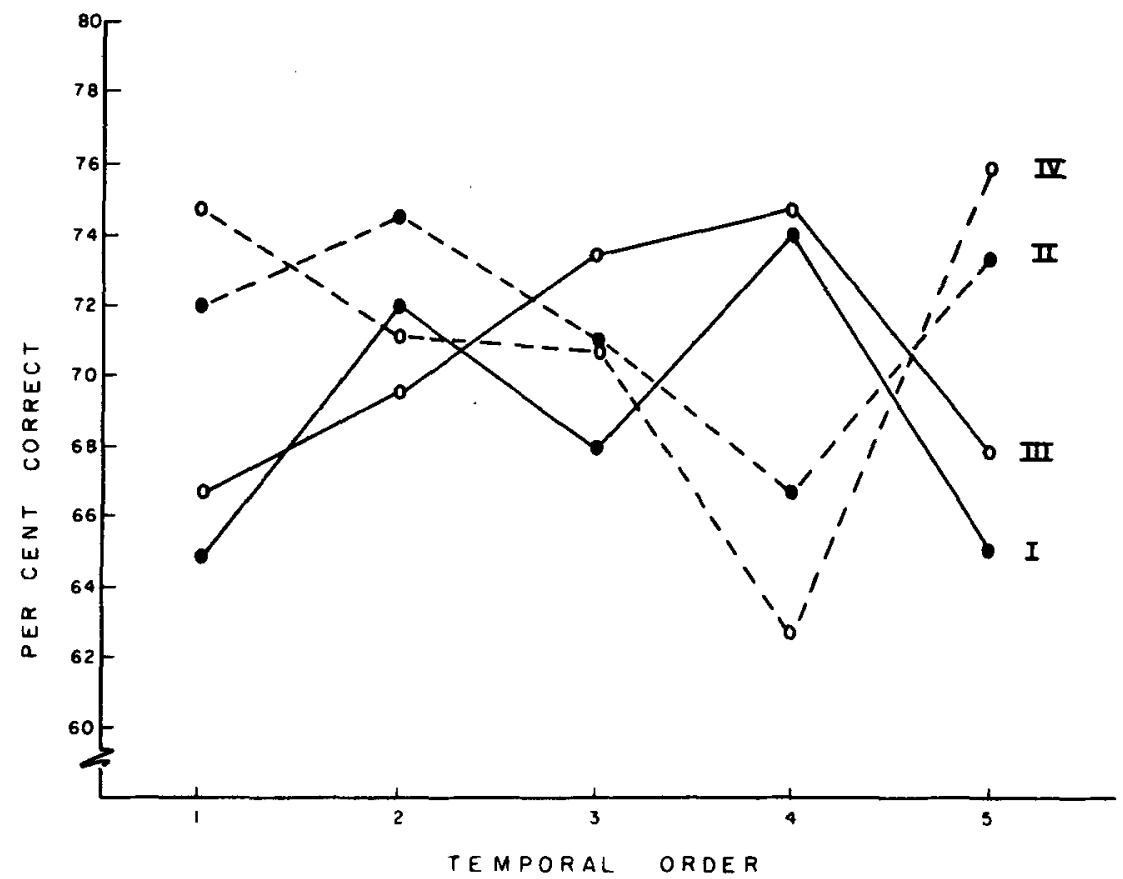

Fig. 2. Per cent correct turget detections as a function of the four spatial locations of the tachistoscopic fields and the temporal onder in which the target occurred. 


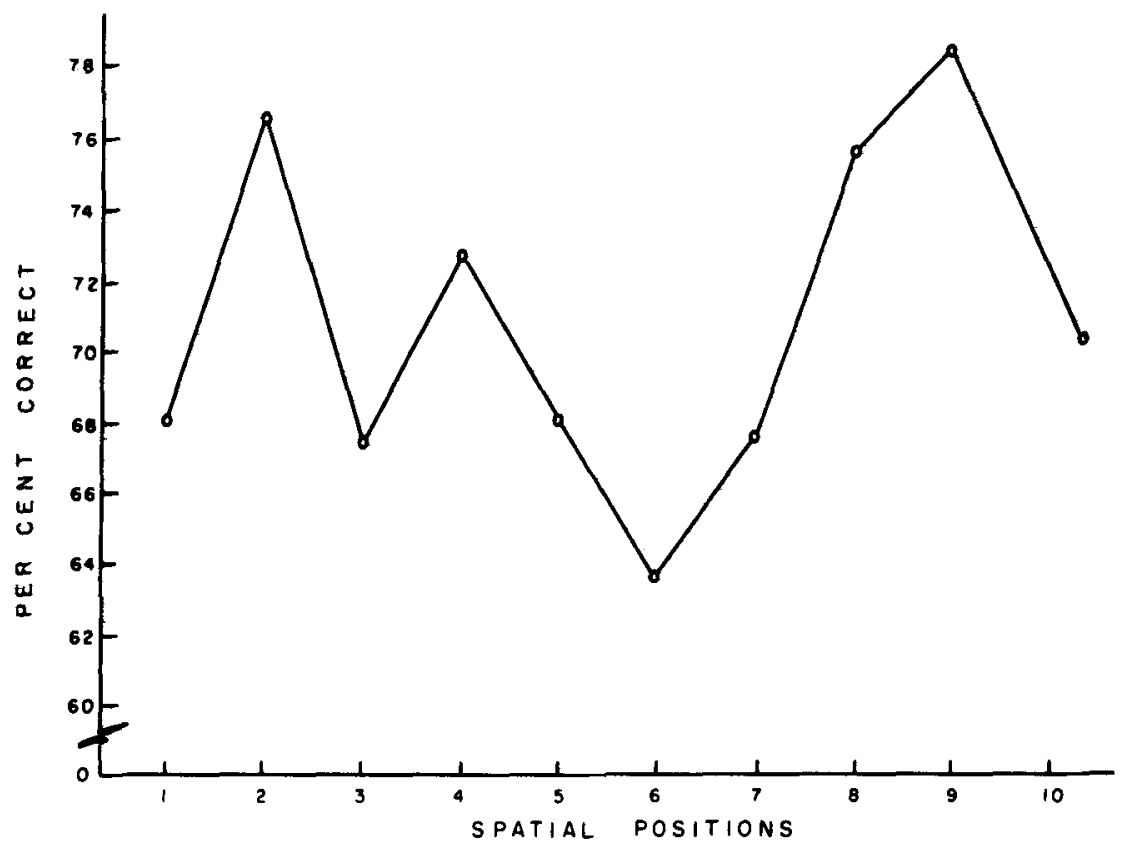

Fig. 3. Per cent correct torget detections in each of the 10 tachistoscopic fields.

choice temporal intervals separated by $500 \mathrm{msec}$, the per cent correct decisions were $89.8 \%$. In the five letter condition the letters were presented within a choice interval at the rate of one letter every $30 \mathrm{msec}$ on the average $(15 \mathrm{msec}$ duration for the letter and $15 \mathrm{msec}$ interletter interval). Here per cent correct was $70.5 \%$ averaged over temporal orders, sequences, and spatial locations. Although this difference between the single and five letter conditions is significant $(t(4)=6.69, p<.01)$ the lower detection accuracy under the five letter condition cannot be unequivocally ascribed to a breakdown in the encoding or processing mechanisms under the high input rate.

There are some inherent differences in the judgmental situation when $S$ has to look at two series of five stimuli and judge which one most likely contained the Target $A$ than when he has to compare only two stimuli to determine which one most likely was an $A$. Even if the $S$ was able to process each of the five letters as efficiently as for the single letter condition lower detection accuracy would be expected for the five letter condition due to the greater opportunity to confuse a noise letter with the target and to a change of what might be termed judgmental strategy. This difference in strategy can be illustrated in the following manner. For the single letter condition the $S$ essentially has two independent chances to perceive the forms. He can make a correct decision if (a) he identified the target when it occurs in one of the intervals, (b) he identifies the $\mathrm{T}$ or the $\mathrm{U}$ noise letter in the other interval, or (c) both of these events occur. If he identifies a $T$ or a $U$ in one of the intervals he knows automatically that the other interval must have contained the target letter irrespective of whether he perceived it when presented.

When $S$ has to decide which of two series of five letters contained the Target $A$ his strategy is not as simple and on the occasions when he fails to perceive the $A$ his probability of chance success is not as great. For example, if he's able to identify three of the letters in one series of five and two of the letters in the other series, none of which are As, his optimum strategy would be to choose the interval in which only two letters were identified since the probability is slightly greater that the $A$ is among the remaining three letters. However, even if $S$ adopts this optimum strategy his probability of choosing the interval that contains the $A$ without actually perceiving or identifying the $A$ is not as high as it is for the single letter condition. This can be proven by assigning a probability value to the perception of any given letter and then computing the probability of identifying one through five of the letters in the sequence in one interval and the corresponding probabilities for one through five letters in the other interval. If the various combinations of probabilities for the two intervals are worked out it will be found that even using optimum strategy the $S$ does not have as high a probability for success where he fails to identify the $A$ as he does under the single letter condition. The judgmental situation becomes even more complex when the probabilities of misperceiving a noise letter as the target are also considered.

While a theoretical solution to these differences in the judgmental task might be possible, our primary interest is in the effect of the $30 \mathrm{msec}$ per letter rate and this question can be answered more simply at the empirical level.

\section{EXPERIMENT 2}

From the conclusions of Sperling (1963) and Scharf, Zamansky, and Brightbill (1966) the $30 \mathrm{msec}$ per letter presentation rate for the five letter condition should have been sufficient for each letter to be processed at the same accuracy level as for the single letter condition. If this is the case and up to five letters can be processed at the rate of 10 to $15 \mathrm{msec}$ per letter, then the observed difference in accuracy between the single and the five letter conditions in Experiment 1 would have to be ascribed to differences inherent in the judgmental tasks under the two conditions. Some information as to whether the $30 \mathrm{msec}$ per letter rate really contributed can be obtained by comparing a single letter condition with a five letter condition where all letters are simultaneously presented. If a rate of 10 to $15 \mathrm{msec}$ per letter is really required for a visual search task, then the performance difference between a single letter condition and a condition in which five letters are simultaneously presented ought to be appreciably greater than that obtained in the previous experiment.

\section{Subjects}

\section{METHOD}

Three Ss from Experiment 1 were able to continue in Experiment 2. Two were unable to do so (one male and one female) and were replaced by a male and femaie $S$ drawn from the same population. Both replacement Ss had served in previous experiments in visual perception.

\section{Procedure}

A two temporal interval forced-choice method was again employed for a single letter and five letter condition. The single letter condition was identical to that of Experiment 1 but in the 
Table 2

Percent Correct Target Detections in the Single and

Five Letter Conditions of Experiment 1 and 2

\begin{tabular}{ccc}
\hline & Experiment 1 & Experiment 2 \\
\hline Conditions & & \\
\hline Single Letter & 89.8 & 84.8 \\
Five Letter & 70.5 & 65.0 \\
\hline Mean Difference & 19.3 & 19.8 \\
\hline
\end{tabular}

five letter condition all five letters in a temporal forced-choice interval were presented essentially simultaneously. Due to limitations in the power supply to the lamps it was necessary to introduce a $.5 \mathrm{msec}$ delay between each of the five letters, but by increasing the luminance in each field from .3 to $1.0 \mathrm{~mL}$ all five letters in a choice interval were presented in a total duration of 10 to $15 \mathrm{msec}$ depending upon the $\mathrm{S}$, a duration comparable to the duration of a single letter in Experiment 1.

For the five letter condition the spatial Positions I, II, IIl, and IV were again employed but only pattern Sequence A was used. Each $S$ received 96 single letter and 160 five letter trials distributed over four experimental sessions. Each session contained two randomized blocks of 12 single letter trials and two randomized blocks of 20 five letter trials. Within blocks the target letter occurred equally often in the first and second interval. Spatial position of the tachistoscopic fields and the order of the trial blocks was counterbalanced within and across sessions over Ss.

Prior to beginning the experimental sessions exposure durations yielding approximately $85 \%$ to $90 \%$ accuracy for the single letter condition were established for each $\mathrm{S}$. These durations ranged from 1.6 to $2.5 \mathrm{msec}$, appreciably shorter than in Experiment 1, due to the increase in the field luminance from .3 to $1.0 \mathrm{~mL}$. Following the establishment of these durations each $S$ was given a practice session before beginning the experimental sessions.

\section{RESULTS}

Table 2 compares the mean forced-choice accuracy for the single and five letter conditions of Experiment 1 and Experiment 2. The performance difference between the single and five letter conditions for the two experiments is almost identical, $19.3 \%$ for Experiment 1 and $19.8 \%$ for Experiment 2. The difference between these differences is of course trivial and insignificant. This finding that the reduction in detection performance is as great between the single and five letter conditions in Experiment 1 as in Experiment 2 indicates that the $30 \mathrm{msec}$ per letter rate of Experiment 1 did not contribute to performance over what would be obtained had the five letters been simultaneously presented.

\section{GENERAL DISCUSSION}

The failure to find a rate of input effect across Experiments 1 and 2 could be attributable to the $30 \mathrm{msec}$ per letter rate being too rapid to permit individual letter processing. Both the $30 \mathrm{msec}$ per letter and the $2-1 / 2 \mathrm{msec}$ per letter rates could be too fast to permit indjvidual letter processing.

An alternative possibility is that processing or encoding in visual perception operates in terms of parallel channels at least for five letters or elements. This interpretation would ascribe the lack of a rate difference as due to the simultaneous parallel processing of five elements under both rate conditions. It would require that the target detection performance difference between the single and the five letter conditions was solely ascribable to the change in the nature of the judgmental task. As was noted above, the possible differences in the nature of the judgmental task from the one to the five letter condition are such that one cannot with certainty determine the performance level to be expected in processing five letters sequentially on the basis of the performance obtained for single letter presentations. A satisfactory resolution of this methodological problem will require the exploration of a wide range of letter jnput rates where the number of letters presented sequentially in a trial or temporal forced-choice interval is held constant across rates so in to mantian comparability of the judgmental task.

The possibility must also be entertained that a visual search task such as employed here und by Estes and associates (Estes \& Taylor, 1964, 1966; Estes \& Wessel, 1966) is not suitable for the study of encoding or processing rates in visual perception. When a search task is employed to study processing or encoding rates the assumption is made that the noise elements or letters are encoded or processed in order to lociste the target. Thus an increase in the number of noise letters results in an increase on the average of the number of elements or letters to be encoded before the target is found. However it may be as Broadbent (1958), Treisman (1964a, $b, c)$, and Bower (1965) have suggested, that filters exist in the perceptual system capable of screening out irrelevant stimuli at a stage prior to encoding. Neisser's (Neisser \& Lazar, 1964; Neisser \& Beller, 1965) work on visual search has suggested parallel channels for search tasks and he has recently proposed (Neisser, 1967) a model of information processing that provides for multichannel feature analyzers and preattentive processes that operate at lower levels in the system prior to the encoding stage.

The possibility of filtering or multichannel preattentive processes that operate under conditions of visual search would also account for the failure to obtain sequential blanking as reported by Mayzner et al (1967) in Experiment 1. In terms of the detection criterion no defect was found for the temporal order in which the target occurred or more importantly, in terms of an interaction of temporal order with the pattern sequence in which the stimuli were presented. However our failure to obtain sequential blanking cannot be solely attributed to the employment of a detection or search task. Inquiry of our Ss as well as observation of the stimulus displays by the Es failed to elicit consistent phenomenal reports of blanking.

There are many other differences in experimental arrangements between the present studies and the experimental arrangement of Mayzner et al (1967), that could well account for our failure to obtain the blanking effect. Differences in procedure such as the use of transilluminated stimuli, the distance between spatially adjacent letters and differences in letter shape are all variables that would effect or reduce the amount of apparent movement experienced in the displays. It is clear, however, that the blanking effect is not a general limitation on perceptual rate nor upon a methodology that depends upon varying sequential rates of visual inputs.

\section{REFERENCES}

ALPERN, M. Metacontrast. J. Opt. Soc. Amer., 1953, 43. 648-657.

BOWER, T. G. R. Visual selection: Scanning versus filtering. Psychon. Sci, $1965,3,561-562$

BROADBENT, D. E. Perception and communication. New York: Pergamon Press, 1958

ESTES, W. K., \& TAYLOR, H. A. A detection method in probablistic modes for assessing information processing from brief visual displays. Proceedings of National Academy of Science, 1964, 2(52), 446-454.

ESTES, W. K., \& TAYLOR, H. A. Visual detection in relation to the display size and redundancy of critical elements. Percept. \& Psychophys., 1966, 1 , 9-16.

ESTES, W. K., \& WESSEL, D. L. Reaction time in relation to display size in correctness of response in forced-choice visual signal detection. Percept. \& Psychophys, 1966, 1, 369-373.

KEELEY, S. M. Visual detection as a function of attentional demand and perceptual system error. Unpublished doctoral dissertation, University of Illinois, 1968.

MAYZNER, M. S., TRESSELT, M. E., \& COHEN, A. Preliminary findings on some effects of very fast sequential input rates on perception. Psychon. Sci., 1966, 6, 513-514.

MAYZNER, M. S., TRESSELT, M. E., \& HELFER, M. S. A provisional model of visual information processing with sequential inputs. Psychon. Monogr. Suppl., 1967, 2, No. 7 (Whole No. 23), 91-108.

NEISSER, U. Cognitive psychology. New York: Appleton-Century Cross, 1967.

NEISSER, U., \& BELLER, H. K. Searching through word lists. Brit. J. Psychol, 1965, 56, 349-358. 
NEISSER, U., \& LAZAR, R. Searching for novel targets. Percept. mot. Skills, $1964,19,427-432$.

SCHARF, B., ZAMANSKY, H. S., \& BRIGHTBILL, R. F. Word recognition with masking. Percept. \& Psychophys., 1966, 1, 110-112.

SPERLING, G. A. A model for visual memory tasks. Hum Factors, 1963, 5 , 19-31.

TREISMAN, A. M. The effective irrelevant material of the efficiency of the selective listening. Amer. J. Psychol, 1964a, 77, 533-546.

TREISMAN, A. M. Monitoring and storage of irrelevant messages in selective attention. J. verbal Learn. verbal Behav., 1964b, 8, 449-459.
TREISMAN, A. M. Selective attention in man. Brit. med. Bull., 1964c, 20 , 12-16.

NOTES

1. This investigation was supported by Public Health Service Research Grant MH-1206 and a Public Health Service Research Career Program Award K6-MH-22,014.

2. Address: Department of Psychology, University of Ilinois, Urbana, Ill. 61801 .

(Accepted for publication June 24, 1968.) 